\title{
Gastrointestinal Cancer. Microbiota and Treatment
}

\section{Gertrudis Adrianza de Baptista*}

Titular Professor at Medicine Faculty Central University of Venezuela, Venezuela

*Corresponding Author: Gertrudis Adrianza de Baptista, Head Professor at Medicine Faculty Central University of Venezuela, Venezuela.

Received: September 13, 2019; Published: November 04, 2019

DOI: 10.31080/ASGIS.2019.02.0095

\section{Abstract}

There are multiple diseases related to nutrition: Anemia, Atherosclerosis, Cancer, Diabetes, Obesity, Hypertension, Parkinson, Cancer is known as a set of ailments characterized by progressive accumulation of mutations of the genome of a cell. The WHO points out that the global incidence of cancer could increase in 2030, up to 21.7 million and 13 million deaths due to population growth and aging. The term microbiota refers to the community of living microorganism's resident in a specific ecological niche. In the human intestine is one of the most densely populated community. In microbiology has found that many modern diseases are related to the microbiota. More than 2000 metagenomic studies have shown that about 540,000 microbe genes have been found in the gut of humans. Intestinal flora has many functions: protective functions, structural and metabolic, e.g. resistance to colonization, carcinogen neutralization, metabolism on bile salts, neurological effects, also influence the ability of the digestive enzymes to act on nutrient molecules, as well as important changes in the Microbiome and Microbiota. "Healthy" individual maintains homeostasis, in disease there is a disturbance. Microbiota alter commensalism: type and time of delivery, antibiotics, medications, genetics, diet, lifestyle, exposure to the environment will lead to metabolic alterations. The prebiotics, probiotics provides nutrients for the host as for the microbes modifying that environment in a favorable or harmful way. The response of the Microbiota expressed by the dietary fiber, as well as the probiotics and symbiotics, correlates with the inflammatory response. The intestinal brain axis and with various pathologies, Gastrointestinal Tract (GIT) molecules controls inflammation of the brain. There are multiple pathways between the Intestinal Microbiota (IM) and the brain, which are bidirectional. Short Chain Fatty Acid (SCFA) are neuroactive metabolites and can modulate the brain and certain diseases. IM is conditioned by variants, as genetics in $30 \%$ and the environment in $70 \%$. These concepts are currently being used both as prevention of multiple diseases and their treatments such as in Cancer, Chemotherapy, Degenerative Neurological diseases, Intestinal diseases, and many more still under study. Verify the microbiota's richness, maintain a healthy diet, be physically active. Probiotics should be administered based on solid scientific evidence. The therapeutic effects are the specific strains and doses dependent. Healthy IM is maintained by consuming a nutritious diet and represents a fundamental pillar in the recovery of cancer patients, important to modulate toxicity. The intervention will be the earliest possible. Nutritional screening and diagnosis of the patient are essential for assertive intervention. Malnutrition is the common comorbidity in cancer patients, with determinants linked to patients, tumor and treatment. Cancer cachexia, chronic form of related disease malnutrition with chronic inflammation, and strongly impairs the prognosis. Sarcopenia is a characteristic of malnutrition. It can appear before weight loss and has a strong negative prognosis effect. Prevalence of loss of muscle mass is $39 \%$, prevalence of malnutrition varies from $25 \%$ to $70 \%$, because of different tools for diagnosis. Challenges in trying to reverse both. Future clinical trials: research dose reductions in sarcopenic patients and dose increase studies based on the evaluation of body composition prior to treatment have the potential to alter cancer treatment paradigms. The mechanisms of microbiota-mediated regulation and innate and adaptive immune responses to tumors, the consequences on the progression of cancer and whether the tumors become resistant or susceptible to different therapeutic regimes against cancer. Increase preclinical evidence on short-term fasting (STF) protects against toxicity and improves the effectiveness of a variety of chemotherapeutic agents in the treatment of various types of tumors.

Keywords: Microbiota; Cancer; Diabetes

\section{Introduction}

"In the Persian version of the Old Testament, it was already pointed out in Genesis that Abraham's longevity was due to the consumption of "sour milk".

"Already in the year 76 after Jesus Christ the Roman historian Pliny recommended the administration of fermented dairy products".
The Nobel Prize winner Elie Metchnikoff in 1908 attributed the longevity of certain Balkan populations to the habitual consumption of fermented dairy products, which contained lactobacilli that "would reduce toxins produced by intestinal bacteria, promoting health and prolonging life" [1].

Citation: Gertrudis Adrianza de Baptista. “Gastrointestinal Cancer. Microbiota and Treatment”. Acta Scientific Gastrointestinal Disorders 2.10 (2019): $11-15$. 
There are multiple diseases related to nutrition, deficit or excess: Anemia, Atherosclerosis, some types of Cancer, Mellitus Diabetes, Obesity, Arterial hypertension, avitaminosis: they are rare in western countries, such as beriberi, rickets, scurvy, pellagra... Inflammatory Bowel Diseases etc. Autism, Depression, Parkinson's, Malnutrition, Bulimia nervosa, Anorexia nervosa, Vigorexia among others.

Cancer is known as a set of ailments characterized by progressive accumulation of mutations of the genome of a cell; there are several homeostatic mechanisms that determine the balance between proliferation and cell death.

The World Health Organization (WHO) points out that the global incidence of cancer could increase by $50 \%$ and reach 15 million new cases by 2020 .

By 2030, the global incidence is expected to increase to 21.7 million cancer cases and 13 million cancer deaths due to population growth and aging. In the USA, Gastric Cancer accounts for more than $20 \%$ of all cases of cancer recently diagnosed, considered the fourth most common type and the second leading cause of cancer death.

Actions on smoking, diet and infections can prevent one third of cancers and that it is possible to cure another third. American Cancer Society Oral Cavity and Oropharyngeal Cancer [2].

It is predicted in Latin America that total cancer mortality will decrease in all countries and in both sexes, except in Argentine women. Cuba had the highest cancer rates in 2019, 136.9/100,000 men and 90.4 women, Mexico showed the lowest, 63.8/100,000 men and 61.9 women. In Cuba in the women, lung cancer rates exceeded those of breast cancer. In 2019, it is predicted that mortality from all neoplasms will decrease by approximately $18 \%$ in Argentina, $26 \%$ in Chile, $14 \%$ in Colombia, $17 \%$ in Mexico and $13 \%$ in Venezuela, corresponding to almost 0.5 million avoiding deaths from cancer. No decrease was observed in Brazil and Cuba Concerns, high rates of lung cancer in Cuba, possible increases in breast cancer in young women and the lack of declines in general in Brazil, Cuba and Venezuelan men [3].

Malnutrition is the common comorbidity in cancer patients, with determinants linked to patients, tumor and treatment. Cancer malnutrition, called cachexia, chronic form of related disease malnutrition with chronic inflammation, and strongly impairs the prognosis. Reduced muscle mass, called sarcopenia, is a characteristic of malnutrition and, therefore, of Cancer Cachexia (CC) that can appear before weight loss and has a strong negative prognosis effect. The prevalence of loss of muscle mass is 39\%. The prevalence of malnutrition varies from $25 \%$ to $70 \%$. For very different detection and diagnostic tools used throughout the world [4].

Today there is "Gold Standard" of excellence in evaluation of body composition, computed tomography, facilitates the understanding of the prevalence of Cancer Cachexia (CC).
Challenges in trying to reverse CC and sarcopenia. Future clinical trials: research dose reductions in sarcopenic patients and dose increase studies based on the evaluation of body composition prior to treatment have the potential to alter cancer treatment paradigms.(Ryan Am, Power D.G Cancer-associated malnutrition, cachexia, and sarcopenia: the skeleton in the hospital closet 40 years later [5].

The mechanisms of microbiota-mediated regulation and innate and adaptive immune responses to tumors, the consequences on the progression of cancer and whether the tumors become resistant or susceptible to different therapeutic regimes against cancer [6].

The study (Marshall K. 2019) provides a complete description of the prevalence of malnutrition due to cancer representative of all treatment settings, types of tumors and stages of the disease. It provides valuable information about cancer malnutrition that allows oncology services to identify opportunities to integrate identification and prevention strategies into care models, resulting in better patient outcomes and lower healthcare costs [7].

Increase preclinical evidence on short-term fasting (STF) protects against toxicity and improves the effectiveness of a variety of chemotherapeutic agents in the treatment of various types of tumors. Preliminary data shows that STF (short-term fasting) is safe but challenging in cancer patients receiving chemotherapy. Clinical research evaluating the potential of STF is in its infancy [8].

The microbiota

- The term microbiota refers to the community of living microorganism's resident in a specific ecological niche. The microbiota resides in the human intestine is one of the most densely populated communities

- A small revolution in microbiology has found that many modern diseases are related to the microbiota [9].

- More than 2000 metagenomic studies have shown that about 540,000 microbe genes have been found in the gut of humans, representing the group of dominant microbes in the ecosystem [10].

- Approximately $55 \%$ of these genes constitute the center of the metagenome (there are genes in at least $50 \%$ of individuals). While the other genes, which appear to be unique, are present in 20\%. However, it has not been possible to grow more than 1,000 species.

\section{Functions of the intestinal flora}

Very frequent mechanisms (common to most):

- Resistance to colonization.

- Production of short chain fatty acids and acidification of the medium (dietary fiber)

- $\quad$ Regulation of gastrointestinal transit.

- Normalization of the microbiota.

- Increased enterocyte regeneration.

- Competitive exclusion of pathogens. 
Common mechanisms common to certain species

- $\quad$ Synthesis of vitamins.

- Direct antagonism against other bacteria.

- $\quad$ Strengthening of the intestinal barrier.

- Metabolism on bile salts.

- $\quad$ Enzymatic activities

- Carcinogen neutralization.

Rare mechanisms specific to different strains

- Neurological effects.

- Immunological effects.

- Endocrinological effects.

- Production of bioactive substances.

Recent studies point to abundant evidence showing the microbiota acting as a metabolic organ, capable of relating to other systems: brain, urogenital system, respiratory tract among others [11], so it also correlates with Parkinson's, Alzheimer's, Autism, Depression diseases, among others.

The response of the Microbiota expressed by the dietary fiber (prebiotics), as well as the probiotics and the combination of pre and probiotics - the symbiotics - correlate with the inflammatory response, the intestinal brain axis and, as well as with various pathologies [1].

Gastrointestinal Tract (TGI) molecules control inflammation of the brain; there are multiple pathways between the intestinal microbiota and the brain, which are bidirectional:

- Endocrines (cortisol),

- Nervosas: Vague. and enteric nervous system.

- Immune (cytokines)

- The hypothalamic-pituitary-adrenal axis and cortisol secretion affects immune cells with changes in the microbiota.

- $\quad$ Alters circulating levels of cytokines with effect on brain function.

- The vague nerve and tryptophan modulation are involved in the relationship of the intestinal microbiota and the brain. AGCC (short chain fatty acids) are neuroactive metabolites 'dietary fiber' can modulate the brain and well-being -serotonin [12].

The intestinal microbiota is conditioned by variants such as genetics: in 30\% and the environment in 70\% (epigenetic) [13].

The Healthy individual, maintains homeostasis (balance), in the disease there is disturbance, healthy eating leads to resistance to infection, metabolic nutrients maintain homeostasis in the TGI Mucosa, Immunity, and so on energy control.

Hence these elements to name are products of the disturbance and disruption of homeostasis:

- Obesity

- Metabolic syndrome
- $\quad$ Diabetes

- Infection

- $\quad$ Early bowel disease

- Metabolic liver disease: NAEH.NASH

- $\quad$ Cancer and others

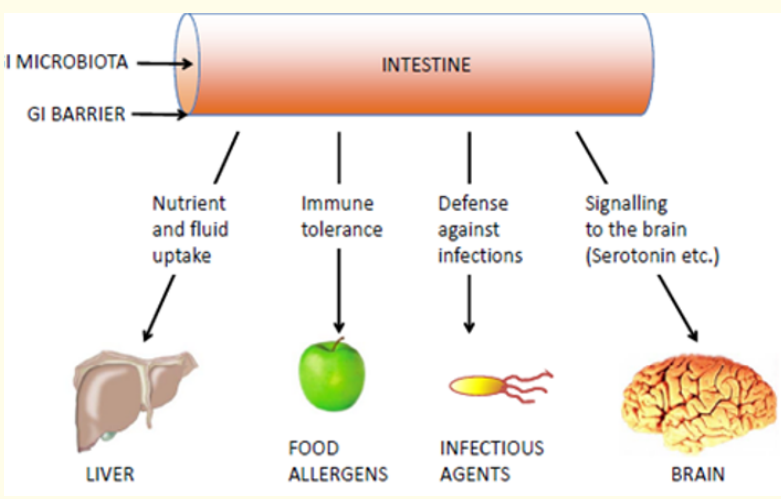

Figure 1: Intestinal barrier without homeostasis is equal to damage to different organs.

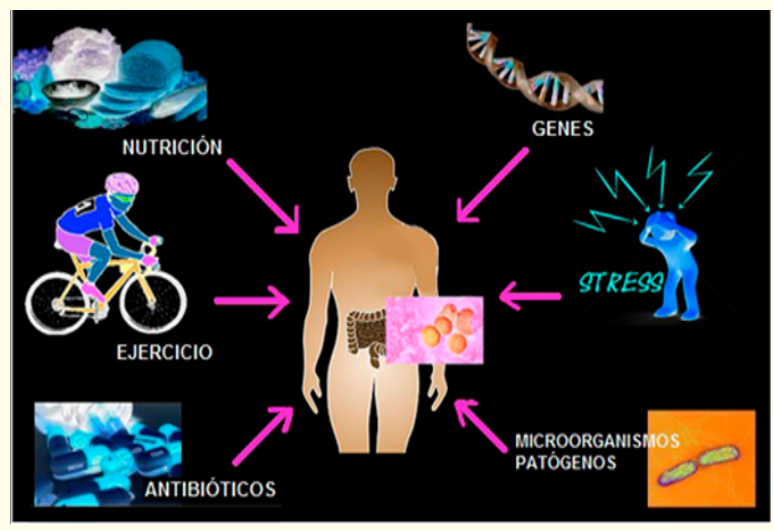

Figure 2: Lifestyle with disturbance = imbalance and equal to the appearance of diseases.

Pregnancy is the biggest biological disturbance. The energy part is complex: needs of the mother and the fetus (in mammals they extend beyond birth). The immune system tolerates fetal growth [14]. All these modifiable elements according to:

- $\quad$ Type and time of delivery (caesarean section or normal)

- Genetics

- Diet

- Exposure to the environment (other humans, animals, medications)

- Health condition.

Liquor, tobacco, drugs, stress among others leads to the loss of commensalism, unlike a healthy life, healthy diet, exercise among others. Microbes play an important role in maintaining health (energy, intestinal protection) and lead to disease. It is lived in commensalism, and homeostasis is broken with pathogenic bacteria.

The microbiota that alter commensalism will lead to metabolic diseases, obesity, IBD (Inflammatory Bowel Disease), cancer and 
others. It increases the presence of harmful bacteria, the diet with fiber- prebiotics- probiotics, symbiotics provides nutrients for the host as for the microbes modifying that environment in a favorable or harmful way.

The microbiota protects the host, increases resistance to pathogens. Donate special vitamins and increase caloric extraction of food. The presence of the microbiota alone means weight gain and energy storage.

\section{Prebiotics}

What are they? Fiber Non- digestible compounds present in the diet, that stimulate the growth or activity of native microorganisms, resulting in health benefits.

Composition: Oligo or Polysaccharides, formed by the polymerization of fructose, galactose and mannose, mixed compounds such as lactulose and lactitol. When added to food, oligo and polysaccharides (broad spectrum prebiotics) are usually combined. Fruits, vegetables, grains.

What effects do they produce? It favors the development of bifidobacteria, as well as the mechanical and metabolic aspects. Many of the bacteria present in their genomes the necessary determinants to degrade various complex carbohydrates, although many firmicutes and, especially, Bacteroides, also have similar microbial genes.

The latest FAO definitions aim to consider the degree of polymerization of substances classified as Fiber. We would have monosaccharides, disaccharides, oligosaccharides, starches and polysaccharides that are not starch.

Soluble fibers influence nutrient absorption. They also influence the ability of the digestive enzymes to act on nutrient molecules, as well as important changes in the Microbiome and Microbiota.

Fiber constitutes the portion of non-digestible plant foods; insoluble fibers (cellulose and lignin) or soluble fructose and galactose oligosaccharides. Soluble nutrient complexes that are not absorbed are fermented.

Glycosylated acidic Hydrolase of bacterial origin degrades the complex of carbohydrates derived from dietary fiber, producing SCFA: acetate, propionate and butyrate.

The evolution of a stable microbiosis depends, on the constant movement of the intestine, merits that the microbiota be renewed in a stable way.

Over nutrition can alter the microbial ecosystem and have severe consequences for the microbiota and the host.

- Produces SCFA, modifies the pH of the colonic content, maintains the microflora, stimulates the normal production of gastroin- testinal hormones, helps improve the intestinal barrier's defenses against bacterial translocation and intervenes on the mucin of the colon and mucus formation.

Mucin and mucus secretion are a viscous secretion, with 95\% water, electrolytes, proteins, nucleic acids, enzymes, immunoglobulins and mucins. Its possibilities are wide:

1. Serves as a substrate for microflora

2. Facilitates microbial destruction, accentuating the defensive barrier

3. It acts as an antioxidant.

4. Modulates the absorption of fatty acids and cholesterol.

The preparation of enteral diets with fiber and bifidobacteria, in adequate proportions both quantitatively and qualitatively (soluble/insoluble), adds a positive value to the indication of these diets in diseases to keep the gastroenteric functionalism and nutrition of the patient safe.

Probiotics are live microorganism intended to provide health benefits when consumed, generally by improving or restoring the gut flora. Live probiotic cultures are part of fermented dairy products, other fermented foods, and probiotic-fortified foods.

\section{Therapy: possible strategies}

- $\quad$ Diet (includes prebiotics/fibers)

- Antibiotics

- Probiotics

- Prebiotics

- $\quad$ Symbiotics (prebiotic + probiotic)

- $\quad$ Fecal Microbiota Transplant for certain types of diseases in patients with Clostridium Difficult in Intensive Care Unit.

All these concepts explained, are currently being used both as prevention of multiple diseases and their treatments, such as, in Cancer, Radiation Enteritis, Chemotherapy, Pancreatitis, degenerative neurological diseases, Intestinal diseases, and many more still under study.

\section{Practical recommendations}

Verify the microbiota's richness: Maintain a healthy diet (e.g. whole grains, fibers, routes and vegetables).

Be physically active
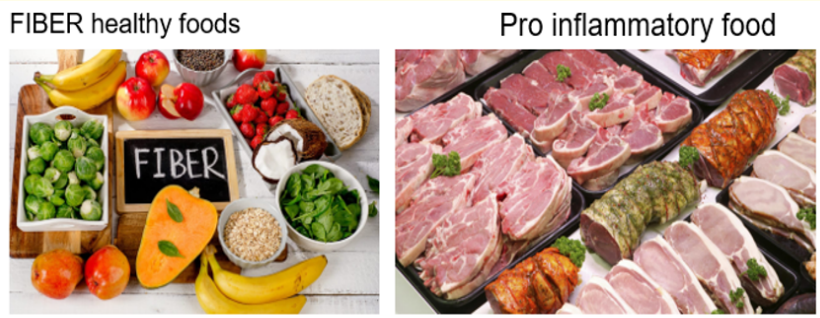

Figure 3 
How to changes the Microbiota?

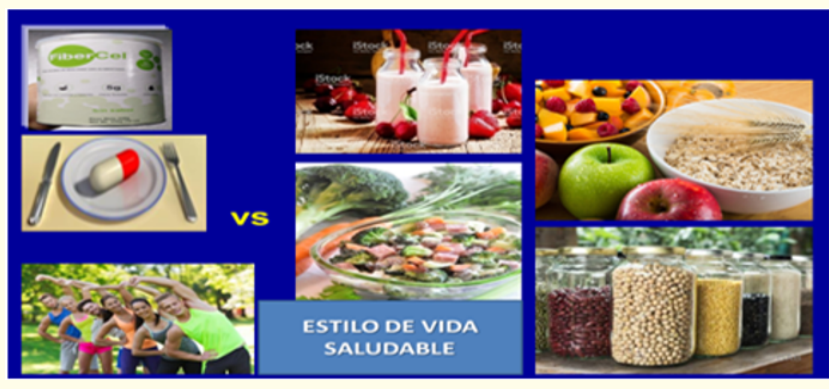

Figure 4

- Probiotics should be administered with care and caution and only based on solid scientific evidence.

- The therapeutic effects are strain specific and dose dependent.

- $\quad$ The best way to maintain a healthy intestinal microbiome is to consume a nutritious diet, varied and rich in fibergrains-fruits-vegetables

- $\quad$ Fecal microbial transplantation is a highly complex and skillful procedure.

- Clearly a new and emerging field.

- Be physically active

\section{Conclusion}

It is clear the microbiota plays a critical role in the performance of the functions of the gastrointestinal system, through the regulation of the inflammatory response and of immune homeostasis $[15,16]$.

Recent studies point to abundant evidence showing the microbiota acting as a metabolic organ, capable of relating to other systems: brain, urogenital system, respiratory tract [11].

The ability of the microbiota to influence fat stores and influence metabolism has also been demonstrated [17]. Verify microbial wealth.

The diagnostic criteria of malnutrition have received a lot of attention in recent years, especially among patients with cancer, the disease population most at risk for malnutrition. Malnutrition in patients with cancer, as in most individuals, is a multifactorial comorbidity that is patient-, disease- and treatment-related, mostly involving a reduced food intake.

The recent Global Leadership Initiative on Malnutrition (GLIM) has become a consensus alternative to standardize how malnutrition may be diagnosed based on etiologic and phenotypic criteria available everywhere, from rich to poor countries. This will lead to an easier diagnosis of cancer malnutrition that itself will help us speak the same language worldwide [4].

\section{Bibliography}

1. Oliveira G and González- Molero I. "An update in probiotics, prebiotics in clinical nutrition". Endocrinology Nutrition 63(2016):482-494.

2. www.cancer.org

3. G Carioli., et al. "Cancer Mortality prediction for 2019 in Latin America”. International Journal of Cancer (2019).

4. Stephane M., et al. "Epidemiology of weight loss, malnutrition and sarcopenia: a transatlantic view”. Nutrition 69 (2020): 110581.

5. Ryan Am and Power DG. "Cancer-associated malnutrition, cachexia, and sarcopenia: the skeleton in the hospital closet 40 years later". Proceedings of the Nutrition Society 75(2016): 199-211.

6. Amiran Dzutsev., et al. "The role of the microbiota in the in flammation, carcinogenesis, and cancer therapy". European Journal of Immunology (2014).

7. Marshall KM. "Prevalence of malnutrition and impact on clinical outcomes in cancer services: A comparison of two time points". Clinical Nutrition 38(2019):644-651.

8. Jiahui Si., et al. "Shisa3 brakes resistance to EGFR-TKIs in lung adenocarcinoma by suppressing cancer stem cell properties". Journal of Experimental and Clinical Cancer Research 38 (2019).

9. Ruiz Alvarez Y., et al. "Intestinal microbiota, Inmune System and Obesity". Revista Cubana de Investigaciones Biomédicas (2012): 29.

10. Qin Ruiqiang Li and Jun Wang. "A Human gut microbial gene catalogue established by metagenomic sequencing". Nature 464 (2010): 59-65.

11. Shader (2012).

12. Wekerle H. "Microglial control of astrocytes in response to microbial metabolites". Nature 557(2018):724-728.

13. http://www.gutmicrobiotaforhealth.com/es/dieta-y-microbiota-intestinal

14. Erlebacher Adrian. "Immunology of the Maternal-Fetal Interface”. 31(2013):387-411.

15. Artis D. "Epithelial cell recognition of commensal bacteria and maintenance of immune homeostasis in the gut". Nature Reviews Immunology 8 (2008): 411-420.

16. Shader A., et al. (2012)

17. Sharon Greenblum., et al. "Metagenomic systems biology of the human gut microbiome reveals topological shifts associated with obesity and inflammatory bowel disease". PNAS 109 (2012): 594-599.

Volume 2 Issue 9 November 2019

(C) All rights are reserved by Gertrudis Adrianza de Baptista. 